\title{
Photoluminescence and Absorption Studies of Defects in CdTe and ZnxCd1-xTe Crystals
}

Cheryl Barnett Davis

cheryl_davis@byu.edu

David D. Allred

allred@byu.edu

\section{A. Reyes-Mena}

Jesus González-Hernández

Ovidio González

Follow this and additional works at: https://scholarsarchive.byu.edu/facpub

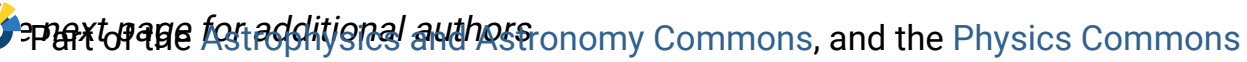

\section{Original Publication Citation}

Cheryl Barnett Davis, David D. Allred, A. Reyes-Mena, Jesus González-Hernández, Ovidio González, Bret C. Hess, and Worth P. Allred, "Photoluminescence and Absorption Studies of Defects in CdTe and ZnxCd1 xTe Crystals," Physical Review B 47(2), 13363-13369 (1993). [http://link.aps.org/doi/1.113/PhysRevB.47.13363][1.113/PhysRevB.47.13363].

\section{BYU ScholarsArchive Citation}

Davis, Cheryl Barnett; Allred, David D.; Reyes-Mena, A.; González-Hernández, Jesus; González, Ovidio; Hess, Bret C.; and Allred, Worth P., "Photoluminescence and Absorption Studies of Defects in CdTe and ZnxCd1-xTe Crystals" (1993). Faculty Publications. 1178.

https://scholarsarchive.byu.edu/facpub/1178

This Peer-Reviewed Article is brought to you for free and open access by BYU ScholarsArchive. It has been accepted for inclusion in Faculty Publications by an authorized administrator of BYU ScholarsArchive. For more information, please contact ellen_amatangelo@byu.edu. 


\section{Authors}

Cheryl Barnett Davis, David D. Allred, A. Reyes-Mena, Jesus González-Hernández, Ovidio González, Bret C. Hess, and Worth P. Allred 


\title{
Photoluminescence and absorption studies of defects in $\mathrm{CdTe}$ and $\mathrm{Zn}_{x} \mathrm{Cd}_{1-x} \mathrm{Te}$ crystals
}

\author{
Cheryl Barnett Davis, David D. Allred, and A. Reyes-Mena* \\ Department of Physics and Astronomy, Brigham Young University, Provo, Utah 84602 \\ J. González-Hernández \\ Centro de Investigación y de Estudios Avanzados del Instituto Politécnico Nacional Unidad Saltillo, \\ Carretera Saltillo-Monterrey Kilómetro 13, Apartado Postal 663, 25000 Saltillo, Coahuila, Mexico \\ Ovidio González and Bret C. Hess \\ Department of Physics, California State University, Fresno, California 93740 \\ Worth P. Allred \\ Galtech Semiconductor Materials Corporation, Mt. Pleasant, Utah 84647 \\ (Received 14 December 1992)
}

\begin{abstract}
We have studied at cryogenic temperatures photoluminescence features which lie more than $0.15 \mathrm{eV}$ below the band edge in $\mathrm{Zn}_{x} \mathrm{Cd}_{1-x} \mathrm{Te}(0 \leq x \leq 0.09)$ crystals. The same features, namely a defect band which lies at about $0.13-0.20 \mathrm{eV}$ below the band-gap energy and a peak at $1.1 \mathrm{eV}$, that are observed in pure CdTe samples are observed in these alloy materials. In annealed samples we observe that the 1.1$\mathrm{eV}$ feature, which has been attributed to tellurium vacancies, increases with fast cooling. Increased concentrations of tellurium vacancies can be understood in terms of the phase diagram of CdTe which indicates that higher concentrations of excess $\mathrm{Cd}$ appear in CdTe quenched from high temperatures. We also observe an absorption transition near $1.1 \mathrm{eV}$ by photothermal deflection spectroscopy (PTDS). The PTDS phase shifts show that the deep defect is a bulk effect rather than a surface effect. The welldefined absorption peak suggests that the states contributing to the 1.1-eV transition are both localized. Our results also suggest that the defect band which lies $0.13 \mathrm{eV}$ below the band gap (1.48 $\mathrm{eV}$ in $\mathrm{CdTe}$ ) may also be related to tellurium vacancies. However, the fact that the ratio of intensities between this defect band and the 1.1-eV feature is highly variable suggests that the relationship is not simple. The origin of the defect band and its phonon replicas remains controversial.
\end{abstract}

\section{INTRODUCTION AND BACKGROUND}

Zinc cadmium telluride $\left(\mathrm{Zn}_{x} \mathrm{Cd}_{1-x} \mathrm{Te}\right)$ is a directband-gap semiconductor with possible applications in near-infrared optical electronic devices such as lasers, photovoltaic and photoconductive infrared detectors, high-efficiency solar-cell structures, and electroluminescent devices. ${ }^{1-3}$

$\mathrm{Zn}_{x} \mathrm{Cd}_{1-x} \mathrm{Te}$ is also a substrate material for the epitaxial growth of mercury cadmium telluride $\left(\mathrm{Hg}_{1-y} \mathrm{Cd}_{y} \mathrm{Te}\right)$. High crystalline quality is needed in its use as a substrate. Defects in the substrate often cause defects in the epitaxial layers. $\mathrm{Zn}_{x} \mathrm{Cd}_{1-x} \mathrm{Te}$ is a good substrate material because its lattice parameters can be matched to those of $\mathrm{Hg}_{1-y} \mathrm{Cd}_{y} \mathrm{Te}$; thus, defects due to "misfit dislocation" are minimized. ${ }^{4-6} \mathrm{Zn}_{x} \mathrm{Cd}_{1-x} \mathrm{Te}$ also could be useful for the formation of superlattices in combination with other IIVI compounds. For these applications, understanding the annealing behavior and identifying and eliminating impurities are essential. This material has a narrow band gap that can be tailored from that of cadmium telluride, $\mathrm{CdTe}$ (1.44 eV at room temperature), to that of zinc telluride, $\mathrm{ZnTe}\left(2.26 \mathrm{eV}\right.$ at room temperature). ${ }^{7}$ It has the same characteristics as other direct-band-gap semiconductors with the added advantage that its band gap ranges from the infrared into the visible; its study as an electronic material is in its infancy.

A large effort has been expended in studying $\mathrm{Hg}_{1-y} \mathrm{Cd}_{y} \mathrm{Te}$ and other similar materials, but $\mathrm{Zn}_{x} \mathrm{Cd}_{1-x} \mathrm{Te}$ has not been investigated to as large an extent. This deficiency is mainly due to the lack of experimental work in the infrared region and the lack of highquality samples. Basic research on the typical photoluminescence spectrum of $\mathrm{CdTe}$ has been done. The spectra for $\mathrm{Zn}_{x} \mathrm{Cd}_{1-x} \mathrm{Te}$ are similar except that the near-gap emission lines are all shifted to higher energies (shorter wavelengths) and the bound-exciton lines are broader than in CdTe. ${ }^{2}$

Other members of our group have previously published studies of $\mathrm{Zn}_{x} \mathrm{Cd}_{1-x} \mathrm{Te}$ in the near-gap region (energies greater than $1.4 \mathrm{eV}) .^{2,3}$ Now we have extended this study down to about $0.7 \mathrm{eV}$. We have studied features in $\mathrm{Zn}_{x} \mathrm{Cd}_{1-x} \mathrm{Te}$ at approximately 1.4 and $1.1 \mathrm{eV}$ which are similar to features studied well in CdTe. In the literature the 1.4-eV feature has been termed the defect band. ${ }^{8-12}$ The peak at $1.1 \mathrm{eV}$ (which we also term the deep-level feature) has long been known and is also probably due to impurities or defects in the sample. ${ }^{13-16}$ The exact nature of these defects remains a matter of controversy, though it is suggested that this feature is connected with tellurium vacancies. ${ }^{10,15,16}$

In this paper we have used photoluminescence (PL) to 
characterize the defect band and $1.1-\mathrm{eV}$ peak in $\mathrm{Zn}_{x} \mathrm{Cd}_{1-x}$ Te samples containing zinc from $x=0$ to 0.09 . We examined the effect of excitation power, sample temperature, and annealing procedures on these peaks in the PL spectra. In addition we employed photothermal deflection spectroscopy (PTDS), which also shows the presence of a defect absorption near $1.1 \mathrm{eV}$.

\section{DESCRIPTION OF EXPERIMENT}

The liquid-helium-cooled, low-temperature photoluminescence system used argon-ion and $\mathrm{He}-\mathrm{Ne}$ lasers for excitation in the range $1-200 \mathrm{~mW}$. All of the spectra shown use the 488-nm argon-ion laser line. Spectra were also obtained using a red $\mathrm{He}-\mathrm{Ne}$ laser to see the effect of increased penetration of light. No spectra are shown in this report for $\mathrm{He}-\mathrm{Ne}$ since no differences were observed. This suggests we are looking at features whose concentration does not change significantly with depth. A highpass filter was employed to remove plasma lines prior to the sample optics. A cylindrical lens was used to shape the beam so that the beam-illuminated area on the sample was about $1-\mathrm{mm}$ wide by $5-\mathrm{mm}$ high. The emitted light passed through two lenses (relay optics) and a 710-nm low-pass filter before entering a SPEX $\frac{3}{4}-\mathrm{m}$, single-grating spectrometer. The filter cut out laser light reflected off the sample. The detector system was a germanium pin detector coupled to a lock-in amplifier and computer. We investigated the luminescence in the near-infrared and infrared regions $(\sim 600$ to $1800 \mathrm{~nm})$. The PL spectra were normalized to the flat response of the spectrometer detector.

The PTDS experiment was performed at room temperature in $\mathrm{CCl}_{4}$, from 0.7 to $1.8 \mathrm{eV}$. Pump light from a tungsten lamp was dispersed by a $\frac{1}{4}-\mathrm{m}$ monochromator and chopped at $17 \mathrm{~Hz}$. The grazing $\mathrm{He}-\mathrm{Ne}$ laser deflection was detected by a Si-position detector and a lock-in amplifier. The spectra were normalized by the incident intensity as measured by a flat-response pyroelectric detector. The deflection amplitude and phase were extracted numerically from spectra at two orthogonal phases.

High-quality $\mathrm{CdTe}$ and $\mathrm{Zn}_{x} \mathrm{Cd}_{1-x} \mathrm{Te}$ single crystals were prepared from the melt using a modified Bridgman method at Galtech Semiconductor Materials, Inc. Some of our samples $(9 \% \mathrm{Zn})$ are ingot ends and are thus lower in crystalline quality. The composition of the $\mathrm{Zn}_{x} \mathrm{Cd}_{1-x}$ Te samples varied from $x=0$ to 0.09 . The stoichiometric composition was accurately determined both by the position of the main exciton line in the lowtemperature photoluminescence spectra and by $\mathrm{x}$-ray fluorescence using a scanning electron microscope with a $\mathrm{Si}$ detector running Z-number absorption fluorescence (ZAF) software. None of the samples were intentionally doped. The samples were cleaved along the (110) surface.

Samples were annealed at $875 \mathrm{~K}$ for $2 \mathrm{~h}$ and were cooled in one of two ways. The samples were annealed in an evacuated (4 to $5 \times 10^{-3} \mathrm{~Pa}$ ) sealed quartz ampoule. The first method of cooling is referred to as fast cooling. This means the samples were removed directly from the Carbolite model CTF tube furnace after the completion of the annealing time. In the second method, termed natural or slow cooling, the furnace was shut off but the sample remained in the center zone of the furnace until it reached room temperature. Cooling took approximately $12 \mathrm{~h}$.

\section{RESULTS}

In Fig. 1 the PL spectrum for energies from 1.0 to 1.8 $\mathrm{eV}$ of pure $\mathrm{CdTe}$ and two $\mathrm{Zn}_{x} \mathrm{Cd}_{1-x} \mathrm{Te}(x=0.03$, $x=0.09$ ) samples measured at $5 \mathrm{~K}$ are shown. The band gap for each is indicated by arrows. The band gaps are, for increasing zinc concentration, $1.61,1.63$, and $1.65 \mathrm{eV}$, respectively. The spectra show three regions: region $I$ is the near-band-gap region; region II is the defect band, about $1.4 \mathrm{eV}$; and region III is the $1.1-\mathrm{eV}$ (deep-level) feature. The near band gap, which is especially clear in pure CdTe, has been well characterized and consists of free and bound excitons and transitions associated with shallow donors and/or acceptors. ${ }^{2,3,17}$ These features decrease in intensity as zinc content increases. In the $9 \%$ $\mathrm{Zn}$ samples these features are not seen. These features in $\mathrm{Zn}_{x} \mathrm{Cd}_{1-x}$ Te samples have been adequately studied by others and are not the focus of this paper. ${ }^{2,3}$

The defect band region occurs in the region between 1.4 and $1.5 \mathrm{eV}$ and has been seen in a large number of studies. $^{8-12,18}$ Features in approximately this energy range in pure CdTe have been attributed to at least two sources, namely cadmium vacancies (centered at $1.41 \mathrm{eV}$ ) or tellurium vacancies (centered at $1.48 \mathrm{eV}$ ). We attribute the defect bands in our as-prepared samples to tellurium vacancies. The intensity of this band increases drastically with zinc content and the peak shifts with the band gap. The maxima of the peaks lie at 1.48, 1.47, and 1.52 for $x=0,0.03$, and 0.09 , respectively, which means that the maxima lie at $0.13,0.15$, and $0.13 \mathrm{eV}$ below the band gap. Also, in most pure CdTe samples, phonon

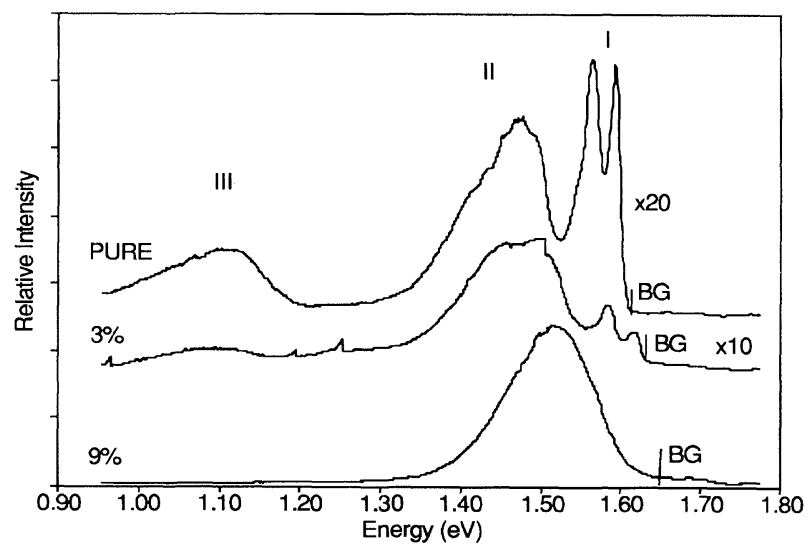

FIG. 1. PL spectrum measured at $5 \mathrm{~K}$ of a pure CdTe and two $\mathrm{Zn}_{x} \mathrm{Cd}_{1-x} \mathrm{Te}(x=0.03, x=0.09)$ samples are shown for energies from 1.0 to $1.8 \mathrm{eV}$. The band gap of each is indicated. The spectra show three regions: I, the near-band-gap region; II, the defect band, about $1.4 \mathrm{eV}$; and III, the 1.1-eV (deep-level) feature. 
replicas (spaced approximately $21 \mathrm{meV}$-typical LO phonon spacing ${ }^{9}$ ) can be seen superimposed on the defect band. These replicas are not seen in higher zinc content samples, however. This is probably due to the substitutional disorder in $\mathrm{Zn}_{x} \mathrm{Cd}_{1-x}$ Te samples. In Fig. 1 the energy resolution is not sufficiently detailed for the phonon replicas to be resolved (see Figs. 3 and 4).

The midgap feature is centered at about $1.1 \mathrm{eV}$. It has a full width at half maximum (FWHM) of about $0.12 \mathrm{eV}$. When the zinc concentration is increased, the intensity of the deep-level peak decreases drastically relative to the defect band but does not increase in energy suggesting its position is not strongly linked to changes in the band gap.

To identify the origin of these features power, temperature, and annealing conditions were varied. In Fig. 2 the spectra of pure $\mathrm{CdTe}$ at five different laser powers are shown. All features in the spectra increase with increasing laser power. The near-gap feature increases more rapidly with power than do the deep-level feature and the defect band. The apparent maximum of the defect band shifts from 1.47 to $1.48 \mathrm{eV}$ with increasing power. This may be due to the increase in the intensity of the neargap feature which partially overlaps with the high-energy side of the defect band. The dependence of the peak intensities of the defect band and the deep-level features on laser power are shown in the inset in Fig. 2. The behavior of these two peaks when laser power is varied is similar.

Figure 3 shows the spectra at three different temperatures. ${ }^{2,10}$ All features decrease in intensity with increasing temperature, particularly those associated with levels near the band gap which are easily ionized. The excitons disappear first followed by the donor-acceptor (DA) transitions. In addition, the individual phonon replica peaks become indistinguishable above about $30 \mathrm{~K}$. Inset $\mathrm{I}$ in

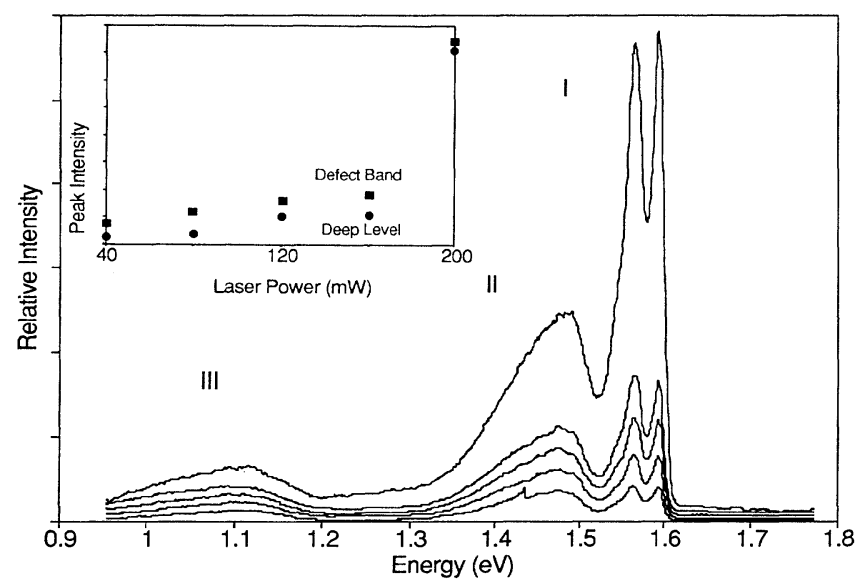

FIG. 2. PL spectra of pure CdTe at five different laser powers, which are $40,80,110,160$, and $200 \mathrm{~mW}$, are shown. All features in the spectra increase with increasing laser power. The near-gap feature (I) increases more rapidly with power than do the other features. The dependence on laser power of the peak intensities of the defect band (region II) and the deep-level (region III of the figure) features are shown in the inset.

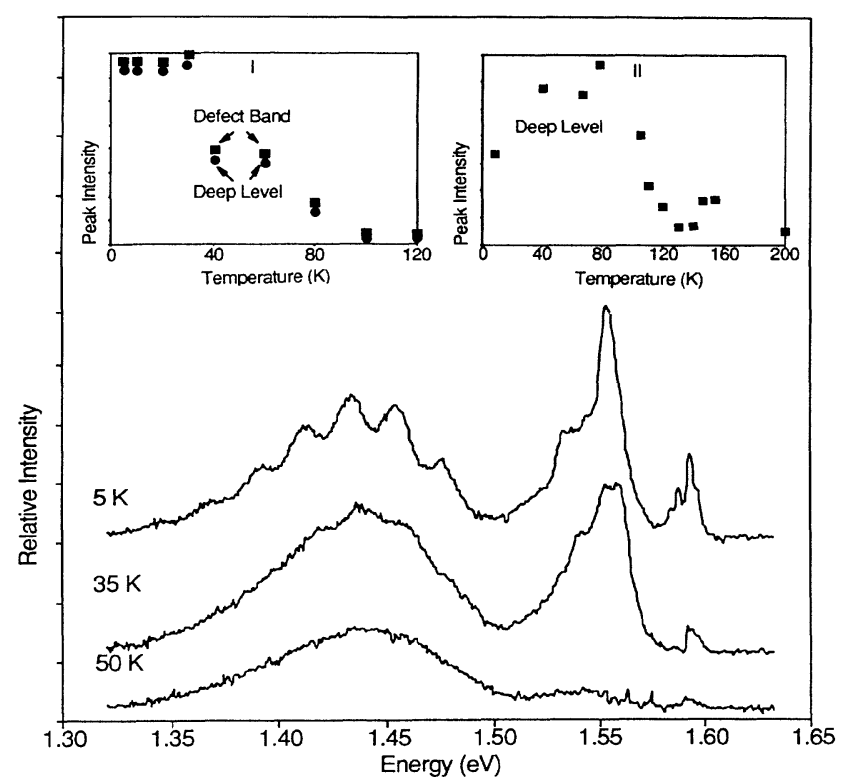

FIG. 3. Temperature dependence of pure CdTe. All features decrease in intensity with increasing temperature. Inset I shows a plot of the peak intensity of the defect band and the deep-level feature vs the temperature in pure CdTe. Inset II shows a plot of the peak intensity of the deep-level feature vs the temperature for $x=0.03$.

Fig. 3 shows a plot of the peak intensity of the defect band and the deep-level feature versus the temperature in pure CdTe (curves 1 and 2). Inset II in Fig. 3 shows a plot of the peak intensity of the deep-level feature versus the temperature for $x=0.03$. The dependence of the defect band on temperature is similar to that of the deep level, but is not shown in the inset. In contrast to pure $\mathrm{CdTe}$, in $\mathrm{Zn}_{x} \mathrm{Cd}_{1-x} \mathrm{Te}(x=0.03)$ the deep-level peak increases to a maximum at $100 \mathrm{~K}$ and then decreases rapidly although not monotonically thereafter.

Figures 4 and 5 show the effect of annealing on various features in $\mathrm{Zn}_{x} \mathrm{Cd}_{1-x} \mathrm{Te}$. Since the samples must be annealed the spectra were collected on the same sample on different days. Differences in noise and intensity are due to instrumental variations from run to run. In Fig. 4 the results of annealing pure $\mathrm{CdTe}$ are shown for energies above $1.2 \mathrm{eV}$. Starting at the band edge and working down, the excitonic features are relatively suppressed in the fast-cooled sample and relatively enhanced in the naturally cooled sample. The opposite trend is seen in the DA region of the spectra.

The most salient feature of the spectra is the defect band. In the first place the peak shifts to high energy for the spectra of the fast-cooled sample. Second, the phonon replicas are not observed at all in the fast-cooled sample and are less pronounced in the slow-cooled sample. The shift in peak position suggests a change in the identity of the defect for the fast-cooled sample. Annealing changes neither the zinc composition nor the band gap, so changes in the defect peak position are attributable to changes in the nature of the defect. 


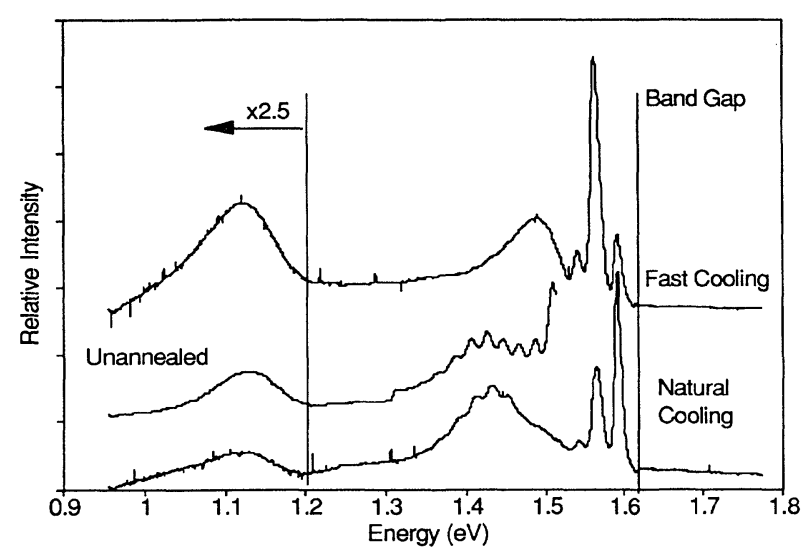

FIG. 4. Annealing data for a pure CdTe sample. Notice the shift of the defect band peak to higher energy in the spectra of the fast-cooled sample. This shift suggests a change in the identity of the defect for the fast-cooled sample. Defect peaks centered near $1.48 \mathrm{eV}(0.13 \mathrm{eV}$ below the band gap) are often associated with $\mathrm{Te}$ vacancies. Those at $1.41 \mathrm{eV}$ are associated with Cd vacancies. Further, notice that the intensity of the deeplevel feature is largest for the fast-cooled sample suggesting a higher concentration of defects.

The deep-level region of these same samples is shown in Fig. 4. Here the intensity of the deep-level feature is largest for the fast-cooled sample followed by the unannealed sample, while it is least intense for the slow-cooled sample.

Figure 5 shows the PL spectra of several 3\% zinc samples; unannealed, naturally cooled, and fast cooled. The most notable feature in these spectra is the defect band. The position of the band and their phonon replicas merit comment. The center of the defect band in the unan-

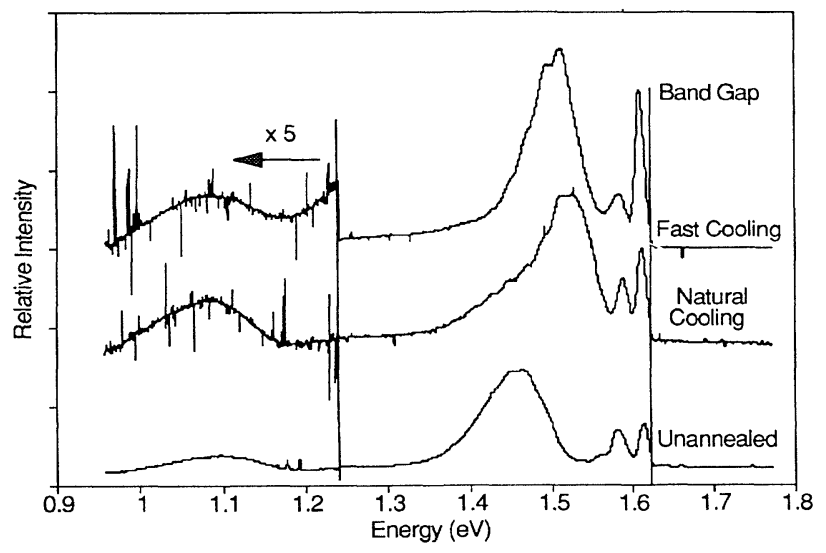

FIG. 5. PL spectra of several $3 \%$ zinc samples: unannealed, naturally cooled, and fast cooled. Notice the shift of the defect band in both of the annealed samples from 1.45 to greater than $1.5 \mathrm{eV}$. This suggests a difference in the phase diagram of pure $\mathrm{CdTe}$ and $3 \% \mathrm{Zn}_{x} \mathrm{Cd}_{1-x} \mathrm{Te}$. Annealing also drastically increases the intensity of the deep-level feature in the spectra of both the fast-cooled and naturally cooled samples.

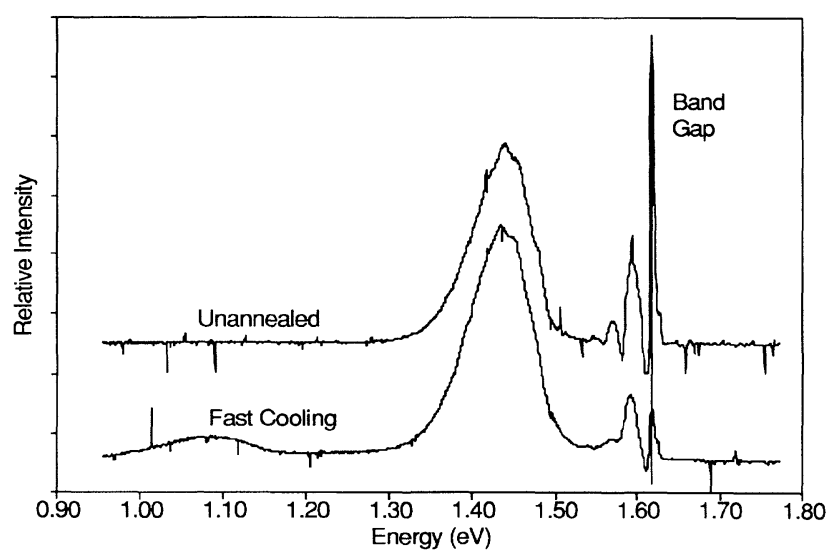

FIG. 6. Spectra of a different $3 \% \mathrm{Zn}_{x} \mathrm{Cd}_{1-x}$ Te sample (lower in crystalline quality) before and after annealing where the fastcooling method was used. In this sample there is no shift seen in the defect band in the annealed sample. Annealing also significantly increases the deep-level peak signal.

nealed sample lies at $1.45 \mathrm{eV}(0.18 \mathrm{eV}$ below the band gap). The naturally and fast-cooled samples lie at 1.52 and $1.49 \mathrm{eV}$ ( 0.11 and $0.14 \mathrm{eV}$ below the band edge), respectively. This is in contrast to pure CdTe where, only in the case of the fast-cooled samples, the defect band lies closer to the band edge.

The phonon replicas are clearest in the naturally cooled sample. In the spectra of the naturally cooled and the fast-cooled samples a train of phonon replicas can be seen on the low-energy side of the peak.

Figure 5 also shows the spectra for these samples below $1.2 \mathrm{eV}$. Annealing drastically increases, and by approximately the same factor, the intensity of the deep-level feature in both the fast-cooled and naturally cooled samples.

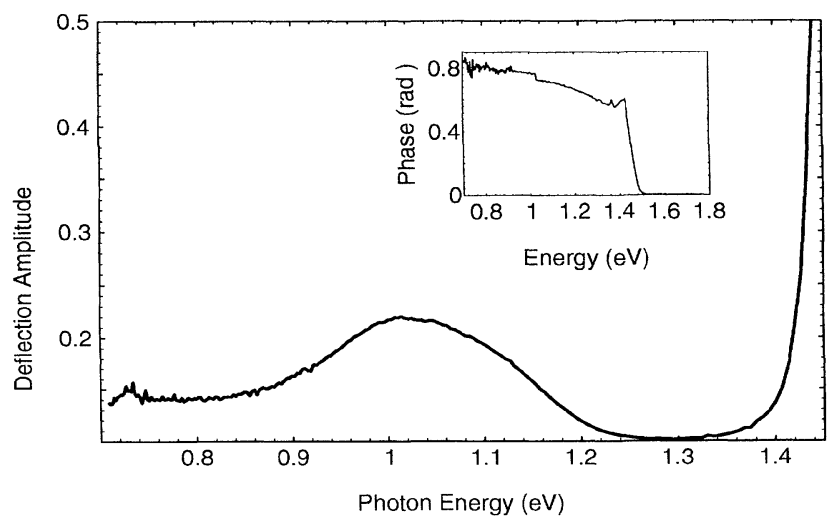

FIG. 7. PTDS spectrum of a fast-annealed pure CdTe crystal, taken at room temperature. The presence of a $1.1-\mathrm{eV}$ peak indicates that it arises from two, rather than one, localized states in the gap. The inset shows the dramatic deflection phase shift between the band-edge absorption and the defect absorption at lower energies. 
Figure 6 shows the spectra of a different $3 \%$ $\mathrm{Zn}_{x} \mathrm{Cd}_{1-x}$ Te sample (taken from the ingot ends and thus lower in crystalline quality) before and after annealing where the fast-cooling method was used. In contrast to previous samples there is no shift seen in the defect band in the annealed sample. Annealing also significantly increases the deep-level peak. The intensity of the exciton and DA peaks follows the same trend as the fast-cooled sample in Fig. 5.

All samples studied by PTDS exhibit a well-defined deep defect absorption peak. We show in Fig. 7 a representative PTDS spectrum of a fast-annealed pure CdTe crystal, taken at room temperature. The peak is near $1.02 \mathrm{eV}$, with a FWHM of $0.2 \mathrm{eV}$. The inset shows the dramatic deflection phase shift between the band-edge absorption and the defect absorption at lower energies.

\section{DISCUSSION}

Figure 1 suggests that the incorporation of zinc into the pure CdTe samples can increase the magnitude of the defect band while relatively decreasing the magnitude of the excitons, DA transitions, and the deep-level peak. In previous work we have studied samples of $\mathrm{Zn}_{x} \mathrm{Cd}_{1-x} \mathrm{Te}$ $(x=0.25)$ of much higher quality showing no evidence of a defect band. ${ }^{19}$

The fact that the intensity of both the deep-level and defect band peaks track with laser power and temperature (Figs. 2 and 3 ) suggests that the origin of the defect band and the deep-level peak in the $\mathrm{Zn}_{x} \mathrm{Cd}_{1-x} \mathrm{Te}$ PL spectrum are related. This is consistent to what has been observed by Sobiesierski, Dharmadasa, and Williams. ${ }^{16}$ But in contrast with their work where they see this correlation between the $1.1-\mathrm{eV}$ peak and the $1.41-\mathrm{eV}$ peak, our defect band peak position is at $1.48 \mathrm{eV}$. There is great controversy about which peaks lie in this region and where their maximum is. Due to the presence of the phonon replica peaks, the maximum is often seen $40-\mathrm{meV}$ lower in energy than the highest-energy peak. Thus, Barnes and Zanio $^{8}$ term this peak the $1.45-\mathrm{eV}$ peak, but the maximum shown in their text is at $1.41 \mathrm{eV}$. Myers et al. ${ }^{18}$ call this peak the $1.42-\mathrm{eV}$ peak.

It is tempting to assume that all peaks in this region $(1.4-1.5 \mathrm{eV})$ are related and result from the same or a similar defect. This is clearly not the case with our samples, however. Compare, for example, the difference between the naturally cooled sample and the fast-cooled sample in Fig. 4. The fast-cooled sample shows a peak centered at $1.48 \mathrm{eV}$ whereas maximum for the naturally cooled sample is at $1.42 \mathrm{eV}$. There is a shoulder in the naturally cooled sample which could be interpreted as a zero phonon at $1.48 \mathrm{eV}$, but the shape of the peak is really much different. The total intensity of the feature is, in addition, only as large as that observed for the fast-cooled sample and the 1.48-eV shoulder is much smaller than the signal at $1.48 \mathrm{eV}$ in the fast-cooled sample. Thus, we can conclude that the process producing light in the two features is somehow different. It is possible that the features in this region (both 1.41 and $1.48 \mathrm{eV}$ ) are due to different defects-one to cadmium vacancies and the other to tellurium defects. These peaks have been previ- ously attributed to either cadmium or tellurium vacancies. In agreement with other authors, however, we conclude that the exact nature of these defects remains unclear.

On the other hand, the 1.1-eV transition has previously been linked to a tellurium defect. ${ }^{13-16}$ The peak is broad and the possibility that it is the sum of several features, some also related to cadmium vacancies, cannot be ruled out. As the density of zinc increases the intensity of the deep-level $(1.1-\mathrm{eV})$ transition decreases. This could be due to either an absence of tellurium defects in the higher zinc samples or more structural defects in these samples which provide an alternate channel for the recombination of electrons and holes which would decrease the deeplevel radiative transitions. The loss of radiative transitions in region $I$ is an indication of decreased crystal quality. The defect band peak does not shift relative to the band edge, and the defect band peak and the deeplevel peak do not seem to increase together. This suggests that these peaks do not have the same origin.

Based on prior identifications of the presence of the 1.1-eV feature with tellurium defects, a decrease in the 1.1-eV feature is due to a decrease in tellurium defects in these samples, all other things being equal. The amount and type of nonstoichiometry allowed in CdTe is known to depend on the temperature at which equilibrium is reached. ${ }^{9}$ As zinc replaces cadmium the phase diagram may be expected to shift slightly and the relative concentration of tellurium and group-II (zinc or cadmium) defects allowed at a given annealing temperature could shift as well. When a sample is cooled rapidly, the concentration of defects frozen into the lattice is determined by the temperature from which the sample is quenched, although there may be diffusion even at room temperature. ${ }^{9}$ When it is cooled slowly there will be some shifts in the concentration of defects and the concentration of defects is characteristic of a lower temperature at which defect diffusion rate becomes negligible. Generally the maximum allowed concentration of defects decreases with decreasing annealing temperature. The $1.1-\mathrm{eV}$ feature can be obtained by $875-\mathrm{K}$ annealing of $3 \%$ zinc samples which showed no feature in the spectrum of a sample as prepared (see Fig. 6) and drastically increases in a sample for which a small $1.1-\mathrm{eV}$ peak could be seen in the as-prepared sample (see Fig. 5). It cannot be concluded that annealing always produces tellurium vacancies because we observe (Fig. 4) that for a pure CdTe sample the 1.1-eV feature which is weakly present in the as-prepared sample decreases in intensity when it is annealed using the natural cooling method but increases when the fast-cooling method is used.

The energy of near-band-gap luminescence features such as excitons and DA transitions increases as the band gap increases, since their energy is the band-gap energy minus a small number which does not vary much as the ratio of zinc to cadmium increases in the crystal. Our data indicate that the defect peak may follow the band gap since it is generally 0.13 or $0.20 \mathrm{eV}$ below the band gap in $\mathrm{Zn}_{x} \mathrm{Cd}_{1-x}$ Te. The behavior of the deep-level feature is not as clear. Since it is broad, a shift of $0.05 \mathrm{eV}$ in the band gap may produce changes in the position of 
the $1.1-\mathrm{eV}$ feature which are difficult to detect. However, our studies suggest that the feature may not move with band gap as fast as the near-band features.

The PTDS spectra of Fig. 7 show that the same transition near $1.1 \mathrm{eV}$ seen in PL also occurs in absorption at $1.02 \mathrm{eV}$. The room-temperature band gap of CdTe is $0.17-\mathrm{eV}$ smaller than the $5-\mathrm{K}$ band gap. It appears that this is the same feature which has been shifted by half the shift in the band-gap energy. The peak at $1.0 \mathrm{eV}$ represents a change in $\alpha$ of about $2 \mathrm{~cm}^{-1}$. The welldefined peak indicates that the transition is between two localized states, in contrast to a transition between a localized state and an extended state (e.g., conduction band) for which a shoulder, rather than a peak, is expected. The PTDS phase shift of the deep-level defect near $1.0 \mathrm{eV}$ is approximately $\pi / 4$ relative to that above the band edge. The $\pi / 4$ shift proves that the defects are dispersed throughout the bulk of the crystal, that is, for depths much greater than the thermal diffusion length (approximately $300 \mu \mathrm{m}$ at $17 \mathrm{~Hz}$ ), and are not due simply to changes near the surface of the crystal. ${ }^{20}$ The fact that no differences are observed between green and related red laser light in the photoluminescence studies also suggests that the features studied relate to bulk rather than surface states. Since CdTe is a direct-band-gap semiconductor, however, both wavelengths of light are absorbed within a few hundred nanometers. On the other hand, the subband gap light used in PTDS measurements penetrates at least a thousand times further, making the case for the bulk origin of the $1.1-\mathrm{eV}$ feature more conclusive.

The feature we are studying by PTDS and luminescence appears not to fit the possible energy-level diagrams presented in earlier literature. In contrast with Bryant and Webster ${ }^{14}$ who saw a shoulder in their photoconductivity measurements at $1.1 \mathrm{eV}$ which suggests transitions involving one localized state and one of the continuous bands, we see no photoconductivity peak in this range. ${ }^{21}$ deNobel $^{22}$ saw in luminescence a feature in Au-doped samples which was both broader than the peak we see and which shifted $(0.2 \mathrm{eV})$ with annealing conditions - unlike our samples. Finally, the data of Garlick $^{23}$ were collected in quite a different way than our case which makes meaningful comparisons impossible. Features in the range of 1.0 to $1.1 \mathrm{eV}$ might arise from structural defects or from various impurities. In light of the high quality (purity and resistivity) of our samples, we suggest that the features we observe are due to structural defects in the crystal rather than to impurities which may account for the features observed by earlier researchers. A more complete model for this deep defect in CdTe and $\mathrm{Zn}_{x} \mathrm{Cd}_{1-x}$ Te will be published elsewhere. ${ }^{21}$

Our annealing data suggest that there is a correlation between the defect band which lies approximately 0.13 $\mathrm{eV}$ below the band gap (1.48 eV in pure CdTe, and 1.51 $\mathrm{eV}$ in $3 \%$ zinc CdTe) and the deep-level feature at $1.1 \mathrm{eV}$. Our thermal treatments which favor the creation of the 1.1-eV feature usually move the defect band from $0.2 \mathrm{eV}$ below the band gap to $0.13 \mathrm{eV}$ below the band gap (from 1.41 to $1.48 \mathrm{eV}$ in pure $\mathrm{CdTe}$ ). In the same spirit, in those samples in which the intensity of the 1.1-eV feature is small to begin with, or decreases, the center of the defect band lies $0.2 \mathrm{eV}$ below the band gap $(1.41 \mathrm{eV}$ in pure CdTe-see Figs. 4 and 5). Since the presence of the 1.1$\mathrm{eV}$ feature has been previously attributed to tellurium vacancies, our results suggest that the defect band, which lies $0.13 \mathrm{eV}$ below the band gap $(1.48 \mathrm{eV}$ in $\mathrm{CdTe})$, is related to tellurium vacancies. The fact that the ratio between this defect band and the $1.1-\mathrm{eV}$ feature is highly variable suggests that the relationship is not simple. While the origin of the $1.49-\mathrm{eV}$ defect band and its phonon replicas remains controversial, the recent work of Meikle and Thompson suggests (in light of Ref. 10) that it is due to tellurium vacancies. ${ }^{24}$ Our work suggests that this is true since the position of the defect band moves to about $1.49 \mathrm{eV}$ in the fast-cooled annealed pure CdTe samples which showed a large 1.1-eV peak (see Fig. 4). The identity of the species that produces the defect band in our samples does not appear to change substantially with zinc alloying since the center of the peak generally stays about $0.13-\mathrm{eV}$ below the band edge.

There are two facts which suggest that the two defects, although perhaps related, do not have the same precise origin: first, the ratio of the defect band intensity to that of the $1.1-\mathrm{eV}$ peak can change by many times; and second, the fact that there are samples (Fig. 6-fast cooled) whose spectra exhibit both the 1.1-eV feature and a defect band about $0.2 \mathrm{eV}$ (rather than $0.13 \mathrm{eV}$ ) below the band edge.

There are also sample-to-sample variations. Both Figs. 5 and 6 show spectra from different $3 \% \mathrm{Zn}$ samples from different ingots. The spectra of the unannealed spectra are similar. The position of the defect band for the fastcooled sample in Fig. 5 is $0.13 \mathrm{eV}$ below the band gap, whereas it lies at $0.19 \mathrm{eV}$ below the band gap in Fig. 6 .

This suggests that these are due to different defects in the different samples even though both were fast cooled from unannealed samples whose spectra were initially quite similar.

\section{CONCLUSIONS}

We have studied low-temperature photoluminescence features which lie more than $0.15 \mathrm{eV}$ below the band edge in $\mathrm{Zn}_{x} \mathrm{Cd}_{1-x} \mathrm{Te}$ samples for $x=0$ to 0.09 . The same features that are observed in pure CdTe samples are observed in these alloy materials. We have annealed samples and we have observed that the $1.1-\mathrm{eV}$ feature, which has been attributed to tellurium vacancies, increases drastically in fast-cooled samples. We can understand this in terms of the phase diagram of CdTe which allows for a large cadmium excess in CdTe for higher quenching temperatures. We also observe an absorption transition near $1.1 \mathrm{eV}$ by PTDS. The PTDS phase shifts show that the deep defect is a bulk rather than a surface effect. The well-defined absorption peak suggests that the states contributing to the 1.1-eV transition are both localized. Our results also suggest that the defect band which lies 0.13 $\mathrm{eV}$ below the band gap $(1.48 \mathrm{eV}$ in $\mathrm{CdTe})$ is related to tellurium vacancies. However, the fact that the ratio between this defect band and the 1.1-eV feature is highly variable suggests that the relationship is not simple. The 
origin of the defect band and its phonon replicas nevertheless remains controversial.

\section{ACKNOWLEDGMENTS}

We acknowledge the assistance of Dr. Matthew C. DeLong, Dr. Zhaohui Lin, and Professor P. Craig Taylor of the Department of Physics and Astronomy of the Univer- sity of Utah for the use of their equipment and many helpful discussions including the interpretation of data. We thank Robert Davis who helped collect the data. We acknowledge Professor Larry Knight of the Center for X-Ray Imaging and the Department of Physics and Astronomy of Brigham Young University. This work was also supported by the College of Physical and Mathematical Sciences of Brigham Young University.
*On leave from Departamento de Física, Centro de Investigación y de Estudios Avanzados del Instituto Politécnico Nacional, 07000, México, Distrito Federal, Mexico.

${ }^{1}$ M. G. Peters, A. L. Fahrenbruch, and R. H. Bube, J. Vac. Sci. Technol. A 6, 3098 (1988).

${ }^{2}$ J. González-Hernández, Elías López-Cruz, D. D. Allred, and Worth P. Allred, J. Vac. Sci. Technol. A 8, 3244 (1990).

${ }^{3}$ Elías López-Cruz, J. González-Hernández, D. D. Allred, and W. P. Allred, J. Vac. Sci. Technol. A 8, 1934 (1990).

${ }^{4}$ J. J. Kennedy, P. M. Amirtharaj, P. R. Boyd, and S. B. Qadri, J. Cryst. Growth 86, 93 (1988).

5 J. D. Murphy, Proc. SPIE 659, 2 (1986).

${ }^{6}$ A. M. Glass, R. D. Feldman, D. W. Kisker, P. M. Bridenbaugh, and P. M. Mankiewich, Proc. SPIE 659, 142 (1986).

${ }^{7}$ J. Steiniger, A. J. Strauss, and R. F. Brebrick, J. Electrochem. Soc. 117, 1305 (1970).

${ }^{8}$ C. E. Barnes and K. Zanio, J. Appl. Phys. 46, 3959 (1975).

${ }^{9} \mathbf{K}$. Zanio, in Semiconductors and Semimetals, edited by R. K. Willardson and A. C. Beer (Academic, New York, 1978), Vol. 13.

${ }^{10}$ C. B. Norris and K. R. Zanio, J. Appl. Phys. 53, 6347 (1982).

${ }^{11}$ C. B. Norris, J. Electron. Mater. 9, 499 (1980).

${ }^{12}$ N. C. Giles-Taylor, R. N. Bicknell, D. K. Blanks, T. H. Myers, and J. F. Schetzina, J. Vac. Sci. Technol. A 3, 76 (1985).
${ }^{13}$ F. J. Bryant and E. Webster, Phys. Status Solidi 21, 315 (1967).

${ }^{14}$ F. J. Bryant and E. Webster, Brit. J. Appl. Phys. 1, 965 (1968).

${ }^{15}$ Z. Sobiesierski, I. M. Dharmadasa, and R. H. Williams, J. Cryst. Growth 101, 599 (1990).

${ }^{16}$ Z. Sobiesierski, I. M. Dharmadasa, and R. H. Williams, Appl. Phys. Lett. 53, 2623 (1988).

17J. García-García, J. González-Hernández, Elías López-Cruz, G. Contreras, and J. G. Mendoza-Alvarez, J. Appl. Phys. 67, 3810 (1990).

${ }^{18}$ T. H. Myers, J. F. Schetzina, S. T. Edwards, and A. F. Schreiner, J. Appl. Phys. 54, 4232 (1983).

${ }^{19} \mathrm{~J}$. González-Hernández, A. Reyes-Mena, Elías López-Cruz, D. D. Allred, and Worth P. Allred, in Properties of II-VI Semiconductors: Bulk Crystals, Epitaxial Films, Quantum Well Structures, and Dilute Magnetic Systems, edited by F. J. Bartoli, Jr., H. F. Schaake, and J. Schetzina, MRS Symposia Proceedings No. 161 (Materials Research Society, Pittsburgh, 1990), p. 33.

${ }^{20}$ A. Mandlis, J. Appl. Phys. 54, 3404 (1983).

${ }^{21}$ O. González, T. Iverson, B. C. Hess, A. Reyes-Mena, David D. Allred, and W. P. Allred (unpublished).

${ }^{22}$ D. deNobel, Philips Res. Rep. 14, 361, 430 (1959).

${ }^{23}$ G. F. J. Garlick, J. Phys. Chem. Solids 8, 449 (1959).

${ }^{24}$ S. G. Meikle and D. A. Thompson, J. Electron. Mater. 16, 157 (1987). 